\title{
Концентрации изотопов гелия в глубинных поровых водах, измеренные минералами кернов сверхглубокой скважины СГ-3
}

\author{
Ганнибал М.А. ${ }^{1}$, Колобов В.В. ${ }^{2}$, Барри П.Х. ${ }^{3}$, Тюн Р.Л. ${ }^{4}$, Тараканов С.В. ${ }^{5}$, Добродеев А.С. ${ }^{1}$, \\ Толстихин И.Н. ${ }^{1}$ \\ ${ }^{1}$ Геологический институт КНЦ РАН, Anатиты, Россия, igor.tolstikhin@gmail.com \\ ${ }^{2}$ Центр физико-технических проблем энергетики Севера КНЦ РАН, Апатиты, Россия \\ ${ }^{3}$ Вудс-Холский Океанографический институт, Вудс-Хол, США \\ ${ }^{4}$ Оксфордский университет, Оксфорд, Великобритания \\ ${ }^{5}$ Лаборатория свойств стекла, Санкт-Петербург, Россия
}

Аннотация. Изотопы Не используются для определения времени изоляции подземных вод от контакта с атмосферой («возраста вод»), что необходимо для решения фундаментальных и прикладных задач гидрологии, таких как выбор мест захоронения токсичных и радиоактивных отходов. Концентрации Не обычно измеряются непосредственно в пробах подземных вод, которые, однако, не всегда могут быть отобраны. В таких случаях было предложено использовать равновесие парциальных давлений гелия, $P_{H e}$ в поровых водах и в доступных для гелия объемах в минералах (далее ДГО, например, флюидные включения в кварце). В этой работе $P_{H е}$ определялось в ДГО зерен кварца, отобранных из кернов Кольской сверхглубокой скважины СГ-3 на глубинах около 10 км. Для этого выполнялись эксперименты по извлечению / насыщению зерен гелием при температурах $350^{\circ} \mathrm{C}$. Оказалось, что ДГО уменьшался в ходе экспериментов (вероятно - вследствие декрипитации включений при нагреве образцов), что приводит к завышенным значениям $P_{H e}$. Для устранения этого эффекта была предложена методика, позволяющая: использовать только стабильные, сохранные в ходе лабораторных экспериментов ДГО; получить корректные значения $P_{H e} \cong 1.5$ атм на глубинах 10 км и оценить кон-

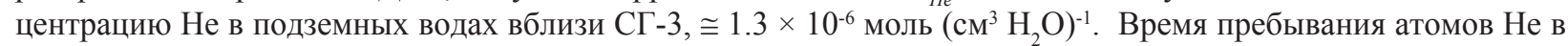
системе вода - порода оказалось близким к возрасту регионального метаморфизма (1700 млн. лет). Отношения ${ }^{3} \mathrm{He} /{ }^{4} \mathrm{He}$ в образцах кварца СГ-3 (и в поровых водах) близки к $3 \times 10^{-8}$, типичным для радиогенного Не, образованного в глубинных архейских породах СГ-3.

Ключевые слова: изотопы гелия, концентрация, подземные воды, кристаллический фундамент, возраст воды.

\section{Concentrations of $\mathrm{He}$ isotopes in deep groundwaters as measured by quartz grains from cores opened by the Kola Superdeep Borehole}

\author{
Gannibal M.A. ${ }^{1}$, Kolobov V.V. ${ }^{2}$, Barry P.H. ${ }^{3}$, Tyne R.L. ${ }^{4}$, Tarakanov S.V. ${ }^{5}$, Dobrodeev A.S. ${ }^{1}$ \\ Tolstikhin I.N. ${ }^{1}$, \\ ${ }^{1}$ Geological Institute KSC RAS, Apatity, Russia, igor.tolstikhin@gmail.com \\ ${ }^{2}$ Center of physical - technical problems of Northern energetic, KSC RAS, Apatity, Russia \\ ${ }^{3}$ Woods Hole Oceanographic Institution, Woods Hole, USA \\ ${ }^{4}$ University of Oxford, Oxford, UK \\ ${ }^{5}$ The Laboratory of Glass Properties, St. Petersburg, Russia
}

\begin{abstract}
Helium isotopes are widely used tracers of water movements and residence times, having important implications for fundamental and applied problems of hydrology, such as radioactive waste disposal. Helium concentrations are generally measured directly in water samples; however, this is not always possible. In these cases, it was proposed to use the equilibrium of the He partial pressure $\left(P_{H e}\right)$ in pore waters and in He accessible volumes (hereafter HAV, e.g., fluid inclusions in a mineral, quartz in this study). The He partial pressure was measured in HAVs of quartz grains collected from deep cores (about $10 \mathrm{~km}$ ) from the Kola Super Deep Borehole (KSDB). To do this, the isothermal extraction / saturation experiments at $350{ }^{\circ} \mathrm{C}$ were applied. A large fraction of He was released from these samples during the first interval of isothermal heating, indicating a destruction of the unstable HAVs, likely due to decrepitation of fluid inclusions. We present a new approach to calculate He partial pressures using only the He fraction released from the stable HAVs. This approach gives a He partial pressure $p_{H e}$ of $\cong 1.5 \mathrm{~atm}$ at depths of $\sim 10 \mathrm{~km}$ and a He concentration in the deep pore waters nearby the $\mathrm{KSDB}, \cong 1.3 \times 10^{-6} \mathrm{~mol}\left(\mathrm{cc}_{2} \mathrm{O}\right)^{-1}$, indicating a long residence time of He atoms in a deep rock - water system, close to the age of the regional metamorphism $(\cong 1700 \mathrm{Ma})$. The ${ }^{3} \mathrm{He} /{ }^{4} \mathrm{He}$ in the deep KSDB quartz samples (and in the pore waters) are $\sim 3 \times 10^{-8}$, typical of radiogenic He generated in $10 \mathrm{~km}$ deep Archean rocks of the KSDB.
\end{abstract}

Keywords: Helium, isotopes, pressure, concentration, deep groundwater, crystalline basement, residence time. 


\section{Введение}

Lehmann et al. (2003) впервые использовали зерна кварца, отобранные из осадочных пород (песчаников) для определения концентраций Не в подземных водах Северной Швейцарии. Впоследствии такой подход (в дальнейшем Метод Равновесных Давлений, МРД) был развит в работах Tolstikhin et al. (2005, 2011, 2018), Gannibal and Tolstikhin (2013), Smith et al. (2013). Парциальное давление Не, $P_{H e}$ в в системе минерал - поровая вода определялось согласно уравнению (1)

$$
P_{H e}=P_{H A C} N_{H e} T_{H e} / N_{H A C} T_{H A C},
$$

где $P, N, T$ - давление, количество Не и температура, а индексы Не и НАС относятся к исходным образцам и к образцам, насыщенным гелием (см.следующий раздел). Работы упомянутых выше авторов позволили определить два важных требования к кварцевым зернам, используемым в МРД. (1) ДГО должны отражать «современную» концентрацию Не в поровой воде: следовательно, равновесие парциальных давлений $P_{H е}$ в поровых водах и в ДГО зерен кварца должно устанавливаться в течение короткого временного интервала (намного меньшего, чем возраст зерен), (2) ДГО должны быть стабильными и не изменяться в ходе экспериментов по экстракции / насыщению гелием используемых образцов.

Зерна кварца, отобранные из осадочных пород Северной Швейцарии, отвечают таким требованиям (Tolstikhin et al., 2011, 2018; Gannibal and Tolstikhin, 2013): образцы содержали захваченный из поровых вод Не; время уравновешивания давлений Не оказалось коротким, 10 000 лет, значительно меньшим возраста пород (285 млн. лет); повторные эксперименты по экстракции / насыщению одного и того же образца не влияли на характер выделения и количество Не, следовательно образцы имели стабильные ДГО. Также было показано, что концентрации Не, полученные МРД, неотличимы от непосредственно измеренных в пробах подземных вод (Tolstikhin et al., 2018).

\section{Образцы кварца из керна Кольской сверхглубокой скважины СГ-3}

СГ-3 была пробурена в докембрийской коре северного Балтийского щита до глубин 12260 м. Скважина пересекает осадочно-вулканогенный комплекс палеопротерозойской Печенгской рифтовой структуры на глубине 6842 м и проникает в архейские породы фундамента, сложенного метавулканическими породами дацитового состава и мета-осадочными гнейсами. Возраст кристаллизации цирконов архейских пород на забое скважины составляет $2830 \pm 10$ млн. лет (Чупин и др., 2009). В палеопротерозое (2.1-1.7 млрд. лет) породы СГ-3 были метаморфированы в условиях амфиболитовой, эпидот-амфиболитовой и зеленосланцевой фации. Использованные в этой работе образцы кварца отобраны из кварцевой жилы и пегматита, встреченных в тектонизированных сегментах разреза СГ-3 с глубин 9860,8 и 11352,2 (далее номера образцов показаны как глубина отбора с коротким названием минерала, кварц в этом исследовании). Зерна кварца содержали многочисленные флюидные включения (потенциальные ДГО).

\section{Эксперименты по экстракции / импрегнации гелия}

Чтобы определить парциальное давление Не в ДГО (уравнение 1), исключив при этом неоднородность образцов, одни и те же зерна кварца последовательно подвергали следующим экспериментам:

(1) Извлечение Не изотермическим нагревом «исходного» (ненасыщенного) образца.

(2) Насыщение того же образца Не.

(3) Извлечение Не изотермическим нагревом насыщенного образца.

Образцы насыщали Не в тигле из нержавеющей стали при давлении Не $P_{H A C}=31$ атм и температуре $T_{H A C}=290^{\circ} \mathrm{C}$ в течение 1008 часов, что обеспечивало полное насыщение. Извлечение из ненасыщенных и насыщенных образцов проводили изотермическим нагревом в тигле из нержавеющей стали, нагреваемом внешней печью до температуры $350^{\circ} \mathrm{C}$ (значительно ниже температуры $\alpha$ - $\beta$ перехода, $573^{\circ} \mathrm{C}$ ). Количество выделившегося Не измеряли с помощью масс-спектрометра MI1201 (Gannibal and Tolstikhin, 2013). 
Для контроля подвижности Не и обеспечения правильного выбора образца Не экстрагировался из зерен кварца при линейном повышении их температуры $\left(1520^{\circ} \mathrm{C}\right.$ в час) с одновременной регистрацией потока Не масс-спектрометром в динамическом режиме.

\section{Время нахождения атомов Не в ДГО образцов кварца}

Для определения времени пребывания Не в зернах кварца, $\tau$, потоки Не из образцов, $d_{t} M$, наблюдаемые при изотермическом и линейном нагреве зерен, аппроксимировались диффузионной моделью, которая связывает поток из сферического зерна (радиус которого принимался равным $r=10^{-3} \mathrm{м}$ ) с коэффициентом диффузии $D$, и количеством Не в зерне $M\left(\right.$ при $\left.t=0, M=M_{0}\right)$ :

$$
d_{t} M=-3 D M / r^{2}
$$

Время пребывания атомов Не в зерне определяется соотношением $\tau=r^{2} / 3 D$ в уравнении (2). Уравнение Аррениуса позволяет связать коэффициент диффузии $D$ с температурой зерна $T, D=D_{0} \exp (-E / R T)$ где $R-$ универсальная газовая постоянная $\left[R=8.31\right.$ Дж моль $\left.{ }^{-1}, K^{-1}\right] ; D_{0}-$ частотный фактор $\left[\mathrm{m}^{2} \mathrm{c}^{-1}\right], E-$ энергия активации [Дж моль ${ }^{-1}$. Параметры $D_{0}$ и $E$ изменялись с целью получения значений, обеспечивающих наилучшее соответствие рассчитанных (из уравнения 2) и наблюдаемых в экспериментах по экстракции Не потоков. Для температуры $T_{H e}=200^{\circ} \mathrm{C}$ (измеренной в стволе СГ-3 на глубине 10 км) получено весьма короткое время пребывания Не в кварцевых зернах $\tau_{200^{\circ} \mathrm{C}} \approx 15$ лет: зерна кварца действительно удовлетворяют требованию (1) в разделе «Введение», независимо от всех неопределенностей, связанных с неточным знанием использованных для расчетов параметров (например, размера и формы диффузионных областей).

\section{Нестабильные ДГО в образцах кварца из СГ-3}

Эксперименты по изотермической экстракции Не из природных образцов показали, что большая фракция Не ( $\Phi_{1}, 0.66$ до 0.76 , см. таблицу) выделяется в ходе первого интервала нагрева длительностью 1 час, а в опытах с насыщенными образцами в первом временном интервале выделяется менее 50 \%. Наиболее простое объяснение этим результатам, - декрипитация некоторых нестабильных флюидных включений при нагреве зерен. Можно показать, что временная шкала нагрева (и декрипитации) весьма коротка. Время прогрева $\tau$ сферического зерна диаметром $\delta$ определяется отношением

$$
\tau=\delta^{2} / a_{T}=\delta^{2} /(\lambda / \rho \mathrm{c})
$$

где $a_{T}$ - коэффициент температуропроводности зерна $\left[\mathrm{m}^{2} / \mathrm{s}\right]$, равный отношению коэффициента теплопроводности $\lambda$ к плотности $\rho$ и удельной теплоемкости $c$. Подставляя в (3) численные значения параметров, характерных для силикатной породы: $\lambda=2 \mathrm{~W} /(\mathrm{m} K), \rho=3 \times 10^{3}$ кг $/ \mathrm{m}^{3}, c=800 \mathrm{~J} /($ кг $K)$, получим время нагрева образца $\sim 1$ секунды. Поэтому только стабильные ДГО разце после первого этапа нагревания и участвовали в: (а) последующих этапах нагревания ненасыщенного (исходного) образца; (б) насыщении этого образца Не и (в) экстракция Не из насыщенного образца. Это объясняет, почему для насыщенных Не образцов СГ-3 наблюдались относительно низкие значения $\Phi_{1}^{*}<\Phi_{l}$.

\section{Парциально давления Не, $P_{н е}$ в стабильных ДГО зерен кварца СГ-3}

Для определения $\mathrm{P}_{\mathrm{He}}$ предположим, что справедливо отношение

$$
\mathrm{He}_{1, \mathrm{CT}} / \mathrm{He}_{2+}=\mathrm{He}_{1} * / \mathrm{He}_{2+} * \text {, }
$$

где $\mathrm{He}_{1, \mathrm{cT}}$ - количество Не, выделившегося из стабильных ДГО мя первого интервала нагрева, $\mathrm{He}_{2+}$ то же, - во время второго и последующих интервалов, $\mathrm{He}_{1}^{*}$ и $\mathrm{He}_{2+}{ }^{*}$ - те же параметры для насыщенного образца. Такое предположение поддерживается данными, полученными для Швейцарских образцов (см. Введение), которые содержали только ДГО $\mathrm{He}_{1, \mathrm{CT}}$ можно выразить через представленные в таблице параметры, $\mathrm{He}_{1, \mathrm{CT}}=\mathrm{He}_{\Sigma} \times(1-\Phi) \times \Phi^{*} /\left(1-\Phi^{*}\right)$; тогда полное количество Не из ДГО 


$$
N_{H e} \equiv \mathrm{He}_{\Sigma, \mathrm{CT}}=\mathrm{He}_{\Sigma} \times(1-\Phi) \times\left\{1+\left[\Phi^{*} /\left(1-\Phi^{*}\right)\right]\right\}=2,36 \times 10^{-9} \text { моль } \Gamma^{-1},
$$

Численное значение в (5) приведено для данных образца 9860.8-кв (таблица), а парциальное давление в ДГО Ст этого образца, найденное из уравнения (1), $P_{H e}=1.68$ атм. В дальнейшем будет использоваться среднее значение $P_{H e} \cong 1.5$ атм (таблица); содержания Не в природных и насыщенных образцах приведены в таблице; другие параметры, входящие в уравнение (1): $T_{H e}=473 \mathrm{~K}, P_{H A C}=31 \mathrm{~atm}, T_{H A C}=570 \mathrm{~K}$.

Таблица 1. Измеренные концентрации, парциальные давления и изотопные отношения в ДГО зерен кварца (образцы 9860-кв и 11352-кв), отобранных из кернов СГ-3.

Table 1. Measured concentrations, partial pressures, and isotope ratios in quartz grains with the effective internal volume of the sample for helium (samples 9860-quartz and 11352-quartz) sampled from the SG-3 drill samples.

\begin{tabular}{|c|l|c|c|c|c|}
\hline $\begin{array}{c}\text { Глубина } \\
\text { отбора, м }\end{array}$ & $\begin{array}{c}\text { Природный } \\
\text { Насыщенный }\end{array}$ & $\begin{array}{c}\text { Содержание } \\
{ }^{4} \mathrm{He}, \text { моль } \Gamma^{-1}\end{array}$ & $\Phi$ & $P_{H e} \mathrm{~atm}$ & ${ }^{3} \mathrm{He} /{ }^{4} \mathrm{He} \times 10^{-8}$ \\
\hline 9860.8 & природный & $5.41 \times 10^{-9}$ & 0.76 & & 5.39 \\
\hline там же & насыщенный & $3.67 \times 10^{-8}$ & 0.46 & 1.68 & \\
\hline 11352.2 & природный & $1.26 \times 10^{-9}$ & 0.66 & & 2.95 \\
\hline там же & насыщенный & $1.32 \times 10^{-8}$ & 0.26 & 1.16 & \\
\hline там же & насыщенный & $1.06 \times 10^{-8}$ & 0.24 & 1.41 & \\
\hline
\end{tabular}

Примечание. Фракции $\Phi \equiv \mathrm{He}_{1} / \mathrm{He}_{\Sigma}-$ для ненасыщенного и $\Phi * \equiv \mathrm{He}_{1} * / \mathrm{He}_{\Sigma}^{*}$ - для насыщенного образца; в числителе - Не выделившийся во время 1-го интервала прогрева, в знаменателе - полное содержание Не (таблица).

\section{Растворимость Не в подземных водах при РТ условиях на глубинах $10 \mathrm{\kappa м}$}

Экспериментальные данные о растворимости Не в воде при литостатическом давлении на глубине 10 км, $P \cong 3000$ атм, и температуре $200^{\circ} \mathrm{C}$ отсутствуют и используются расчетные значения коэффициента Генри $\mathrm{K}_{\mathrm{He}}$ [кг атм моль ${ }^{-1}$, зависящие от температуры и солености (Smith and Kennedy, 1983; Fernández-Prini et al., 2003):

$$
\mathrm{K}_{\mathrm{He}}=174.6 \times \mathrm{Kh} \times \exp \left(\mathrm{S} \text { кг атм моль }{ }^{-1} \mathrm{~K}_{\mathrm{S}}\right),
$$

где $\mathrm{Kh}$ - коэффициент Генри без учета солености, $\mathrm{S}$ - соленость и $\mathrm{K}_{\mathrm{S}}-$ коэффициент Генри для коррекции солености. Численные значения параметров рассмотрены в работе Gannibal et al. (2020). В зависимости от принятых значений солености, - наиболее плохо определенного параметра, значения $\mathrm{K}_{\mathrm{He}}$ варьировали в пределах $800<\mathrm{K}_{\mathrm{He}}<2100$; в дальнейшем использовано среднее значение 1450 кг атм моль ${ }^{-1}$, пересчитанное в размерности коэффициента Бунзена $\beta_{\text {не }}=1 / 1000$ $\mathrm{K}_{\mathrm{He}} \cong 8.5 \times 10^{-7}$ моль см $\mathrm{cm}^{-3} \mathrm{H}_{2} \mathrm{O}$ атм$^{-1}$.

\section{Концентрации Не и отношения ${ }^{3} \mathrm{He} /{ }^{4} \mathrm{He}$ в водах в разрезе скважины $\mathrm{C \Gamma}-3$}

Концентрации Не в глубинных поровых водах определяются как произведение $\mathrm{He}_{\text {пв }}=\beta_{\mathrm{He}} \times \mathrm{P}_{\mathrm{He}} \cong 1.3 \times 10^{-6}$ моль см с $^{-3} \mathrm{H}_{2} \mathrm{O}$. Это значение является одним из самых высоких для природных вод земной коры. Такое количество радиогенного Не могло возникнуть в гнейсах, мигрировать из пород в поровые воды и накопиться в них за время равное возрасту метаморфизма (Gannibal et al., 2020). Представление о локальных источниках Не поддерживается данными об его изотопном составе. Из изложенного выше следует, что отношения ${ }^{3} \mathrm{He} /{ }^{4} \mathrm{He}=(3.1 \pm 1.5) \times 10^{-8}$ (среднее значение для 10 образцов), измеренные в образцах кварца, являются репрезентативными для поровых вод на глубинах $\cong 10$ км вблизи СГ-3. Породы на этих глубинах содержат Не с весьма близким значением ${ }^{3} \mathrm{He} /{ }^{4} \mathrm{He}=(3.5 \pm 1.5) \times 10^{-8}($ среднее для 22 образцов $)$. Анализ U-Th-Не системы говорит о том, что в некоторых породах (гнейсах) возникает достаточно много радиогенного Не, который мигрирует в поровые воды и далее в ДГО кварца и, вероятно, некоторых других минералов.

Работа была поддержана грантом РФФИ 18-05-7004 «Ресурсы Арктики». 


\section{Литература}

1. Gannibal M., Tolstikhin I., 2013. Sandstone minerals as indicators of He residence time in a rock - groundwater system. In: Razerouni, A. M. (Ed.), Sandstone: geochemistry, uses, and environmental impact. Nova Publishers, New York, P. 1-37.

2. Chupin V. et al. Magmatic inclusions in zircons from Archean «grey gneiss» from the Kola Super Deep Borehole as indicator of genesis and age of the protolithes // In: Isotopic systems and time of the geological processes. 2009. Proceedings of the IV Russian Conference on Isotope Geochronology. Saint Petersburg, IGGD RAN, P. 266-268.

3. Fernández-Prini R. et al. Henry 's constants and vapor-liquid distribution constants for gaseous solutes in $\mathrm{H}_{2} \mathrm{O}$ and $\mathrm{D}_{2} \mathrm{O}$ at high temperatures // Journal of Phys. and Chem. Ref. Data. 2003. V. 32. P. 903-916.

4. Lehmann B. et al. Helium in solubility equilibrium with quartz and porefluids in rocks - A new approach in hydrology // Geophys. Res. Lett. 2003. V. 30. P. 1128-1132.

5. Smith S.P., Kennedy B.M. The solubility of noble gases in water and in $\mathrm{NaCl}$ brine // Geochim. Cosmochim. Acta. 1983. V. 47. P. 503-515.

6. Smith S. et al. Testing He equilibrium between quartz and pore water as a method to determine pore water He concentrations // Appl. Geochem. 2013. V. 35. P. 187-195.

7. Tolstikhin I. et al. Helium transfer from water into quartz crystals: A new approach for porewater dating // Earth Planet. Sci. Lett. 2005. V. 238 P. 31-41.

8. Tolstikhin I., Waber H.N., Kamensky I., Loosli H.H., Skiba V., Gannibal, M. Production, redistribution and loss of helium and argon isotopes in a thick sedimentary aquitard-aquifer system (Molasse Basin, Switzerland). Chem. Geol. 2011. V. 286. P. 48-58.

9. Tolstikhin I., Tarakanov S., Gannibal M. Helium diffusivity and fluxes from a sedimentary basin (PermoCarboniferous trough, Northern Switzerland). Chem. Geol. 2018. V. 486. P. 40-49. 